\title{
First Results of XENON100
}

\author{
Marc SCHUMANN* \\ For the XENON100 collaboration. \\ Physik Institut, University of Zurich \\ E-mail: marc.schumann@physik.uzh.ch
}

The XENON100 Dark Matter experiment, installed in the Laboratory Nazionali del Gran Sasso (LNGS, Italy), is searching for WIMP type Dark Matter particles scattering off a $62 \mathrm{~kg}$ liquid xenon target in a dual phase (liquid/gas) time projection chamber. Careful material selection, a novel detector design, and an upgrade of the passive shield, together with capitalizing the self-shielding power of liquid xenon, are crucial in order to achieve a background of less than 0.01 events $\mathrm{kg}^{-1} \mathrm{keV}^{-1} \mathrm{day}^{-1}$ in the fiducial volume. This background, which has been verified experimentally, is lower than in any other dark matter experiment.

The analysis of 11.2 live days of background data taken during a commissioning run in fall 2009 leads to the first science result of XENON100: No events are observed in a pre-defined fiducial volume of $40 \mathrm{~kg}$ mass, excluding spin-independent WIMP-nucleon scattering cross sections above $3.4 \times 10^{-44} \mathrm{~cm}^{2}$ (at $100 \mathrm{GeV} / c^{2}$ ). Below $80 \mathrm{GeV} / c^{2}$, this is the most sensitive exclusion limit so far, constraining the interpretation of DAMA and CoGeNT being due to spin-independent, elastic interactions of light mass WIMPs.

Identification of Dark Matter 2010-IDM2010

July 26-30, 2010

Montpellier France

\footnotetext{
* Speaker.
} 


\section{Introduction}

Various indirect astronomical observations at all cosmological length scales suggest that the vast majority of the energy content of the Universe is dark, i.e. invisible over the whole electromagnetic spectrum. Precise measurements of the anisotropies in the cosmic microwave background show that only about $4 \%$ of the Universe is made from baryonic matter, $21 \%$ is Dark Matter, and $74 \%$ is Dark Energy [1]. While the nature of the Dark Energy remains speculative, there are strong indications that Dark Matter is made up from heavy, non-relativistic (cold) particles that build large scale cosmological structures. Since no Standard Model particle has the right properties to be the Dark Matter particle, it must be from new physics and many experiments aim to find this particle. A well motivated candidate is the WIMP (Weakly Interacting Massive Particle), a stable particle arising naturally in many theories beyond the Standard Model, such as supersymmetry or theories with extra dimensions [2].

There are three general approaches to search for Dark Matter: The first is to create the particles in high energy collisions. The second is to search for indirect astronomical evidences such as gamma-lines from Dark Matter annihilation. In the following we will focus on the third method, the direct detection of WIMPs scattering off target nuclei in the laboratory.

Because they are neutral, WIMPs are expected to interact with the target nucleus (nuclear recoil interactions) whereas the main background comes from electromagnetic interactions of gammas and electrons with the atomic electrons (electron recoil interactions). From the expected WIMP mass and the velocity distribution one expects to measure a steeply falling, featureless nuclear recoil spectrum at energies of a few $\mathrm{keV}$ only. The rates of these interactions are expected to be tiny, much less than one interaction per $\mathrm{kg}$ and day of exposure. Therefore, the experiments have to decrease their background as much as possible in order to be sensitive to WIMPs.

The XENON collaboration uses liquid xenon (LXe) as target material. Xenon is a heavy target $(A \sim 131)$ which enhances the sensitivity for spin-independent WIMP-nucleon scattering. Being an efficient scintillator $(\lambda=178 \mathrm{~nm})$, xenon also serves as detector material. It's high density $\left(\rho \sim 3 \mathrm{~g} / \mathrm{cm}^{3}\right.$ ) allows to build compact detectors with excellent self shielding capabilities. Furthermore, xenon has no long-lived radioactive isotopes, and small admixtures of the radioactive

${ }^{85} \mathrm{Kr}$ can be removed to the ppt level [3].

\section{The XENON100 detector}

Liquid xenon ( $\mathrm{LXe}$ ) detectors provide 3-dimensional interaction vertex reconstruction and signal to background discrimination when operated as a dual-phase (liquid/gas) time projection chamber (TPC), see Fig.1: An interaction in the LXe generates prompt scintillation light (the S1 signal) and ionizes the target. The ionization electrons are drifted towards the liquid gas interface by a strong drift field. Here, the electrons are extracted into the gas phase and accelerated towards the anode while generating proportional scintillation light (S2). Both signals, S1 and S2, are detected by two arrays of photosensors, one immersed in the liquid for optimal light collection, and one located in the gas phase above the target. The position of the interaction can be reconstructed using the $\mathrm{S} 2$ signal distribution on the top array $(x, y)$ and the time difference between prompt $\mathrm{S} 1$ and delayed S2 signal $(z)$. Due to their different track densities in the medium, the ratio S2/S1 


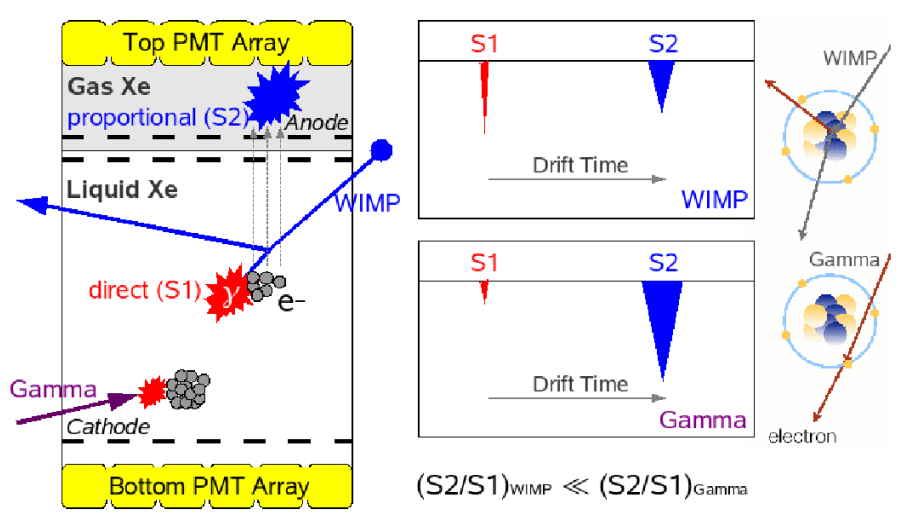

Figure 1: General working principle of a double-phase liquid xenon time projection chamber (TPC): An interaction generates prompt scintillation light (S1) and ionizes the target. The ionization electrons are drifted upwards and extracted into the gas phase where they generate proportional scintillation (S2). The S2 light pattern on the top photosensor array, together with the drift time of the electron cloud, can be used to reconstruct the event vertex in $(x, y, z)$. The ratio $\mathrm{S} 2 / \mathrm{S} 1$ acts as a discrimination parameters as it is different for electron recoil interactions (background) and nuclear recoil interactions (signal).

can be used to discriminate between signal (nuclear recoil interactions) and background (electronic recoils).

XENON100 is the current detector at the $100 \mathrm{~kg}$ scale within the phased program of the XENON collaboration. It follows the XENON10 phase which has proven that liquid xenon detectors are able to deliver very competitive results [4]. XENON100 has a total mass of $161 \mathrm{~kg}$ of LXe, out of which $62 \mathrm{~kg}$ are in the cylindrical target volume which is viewed by two arrays of Hamamatsu R8520 photomultipliers from above (98 PMTs) and below (80 PMTs). The remaining $99 \mathrm{~kg}$ of LXe are surrounding the target in $4 \pi$. This volume is instrumented with another 64 PMTs and acts as an active veto.

The detector, made from low-radioactivity stainless steel, is enclosed by a passive shield made from $5 \mathrm{~cm}$ of ultra-pure OFHC copper, followed by $20 \mathrm{~cm}$ of polyethylene, $5 \mathrm{~cm}$ of low radioactivity lead, and another $15 \mathrm{~cm}$ of standard lead. The whole shield sits on a $20 \mathrm{~cm}$ thick slab of polyethylene and is additionally shielded against neutrons with $\sim 20 \mathrm{~cm}$ of water on four sides. All parts with a potentially higher intrinsic radioactivity, such as the pulse tube refrigerator (PTR) to cool the xenon down to $-92^{\circ} \mathrm{C}$ and the feedthroughs, are placed outside the shield.

\section{Data Analysis and Result}

XENON100 is installed underground at Laboratori Nazionali del Gran Sasso (LNGS, Italy) since spring 2008. After extensive calibrations and studies to characterize the detector response, first science data has been taken in fall 2009. This article summarizes the results of this run, which have been published in [5].

Detector Calibration The response of the PMTs is calibrated by stimulating single photoelectron (PE) emission using blue LED light. The PMT gains were measured regularly and were found to be stable in time within $\pm 2 \%(\sigma / \mu)$. The standard gamma line used to obtain the nuclear recoil energy 
scale from quenching factor measurements (see below) is from ${ }^{57} \mathrm{Co}$ at $122 \mathrm{keV}$. Since $122 \mathrm{keV}$ gamma rays cannot penetrate deep enough into the detector, the average light yield at this energy was derived to be 2.20(9) PE/keV from gamma calibrations with several lines of higher and lower energy: $40 \mathrm{keV}$ and $80 \mathrm{keV}$ from inelastic neutron scattering processes, $164 \mathrm{keV}$ and $236 \mathrm{keV}$ from neutron activated xenon, and with the higher-energy lines from ${ }^{137} \mathrm{Cs}$ and ${ }^{60} \mathrm{Co}$.

Because of the geometry of the TPC, the light (S1) and the charge signal (S2) have to be corrected according to their position in the detector. The correction functions have been measured with different sources at various energies, with a mutual agreement of better than $3 \%$. The largest correction for the S2 signal is due to the finite electron lifetime in the LXe: while drifting towards the liquid-gas interface, a certain fraction of ionization electrons is lost to electronegative impurities in the LXe. The charge loss is described by an exponential function with the electron lifetime $\tau$ as the main parameter. The data presented here were taken with a maximum lifetime of $\tau \sim 200 \mu \mathrm{s}$.

The drift time of the electrons is used to determine the z-position of the interaction. It is measured with a resolution better than $2 \mathrm{~mm}$, limited by the possibility to separate two independent S2 peaks. For the $x y$-position, three independent position reconstruction algorithms (neural network, support vector machine, minimal $\chi^{2}$ ) yield consistent results for $r<140 \mathrm{~mm}$. The precision is better than $3 \mathrm{~mm}$ as verified experimentally using a collimated gamma source.

In order to use the $\log _{10}(\mathrm{~S} 2 / \mathrm{S} 1)$ parameter to discriminate between electron and nuclear recoils, the low energy response of the detector to these interactions has to be calibrated. This has been done using the Compton continuum of ${ }^{60} \mathrm{Co}$ and elastic neutron interactions from an ${ }^{241} \mathrm{AmBe}$ source, respectively. The two populations are well separated and lead to a discrimination of $>99 \%$ at 50\% nuclear recoil acceptance. This measurement is also used to define the WIMP search region in $\log _{10}(\mathrm{~S} 2 / \mathrm{S} 1)$ space: The upper bound is given by the nuclear recoil median, the lower bound by a software threshold which requires the $\mathrm{S} 2$ signal to be $>300 \mathrm{PE}$.

Background All materials considered for the XENON100 detector and the shield were screened in high purity germanium spectrometers in order to determine their intrinsic radioactivity. Only materials with a reasonable radioactivity were accepted for the detector construction. "Hot" components were placed outside the shield. The detector cavity is constantly purged with boil-off nitrogen in order to avoid radon penetrating the shield. All measured radioactivity values are used as input parameters for a detailed Monte Carlo model of the detector and the result of the simulation agrees remarkably well with the measured background spectrum over the full energy range.

In a fiducial volume of $30 \mathrm{~kg}$ mass, XENON100's background rate in the low energy range is $8 \times 10^{-3}$ events $\mathrm{keVee}^{-1} \mathrm{~kg}^{-1} \mathrm{day}^{-1}$ (electron recoil equivalent energy) when the active LXe veto is employed. This is indeed a factor 100 lower than XENON10, thus achieving one of the design goals of XENON100. In fact, XENON100 is currently the experiment with the lowest background level of all running dark matter detectors, more than two orders of magnitude below any other experiment at low energies.

Below 200 keVee, in the energy range of interest, the XENON100 background is dominated by ${ }^{60} \mathrm{Co}$ mainly from the PMTs and ${ }^{85} \mathrm{Kr}$. The latter has been removed from the LXe by cryogenic distillation [3], and the remaining concentration in this run has been measured to be around $150 \mathrm{ppt}$ via a delayed coincidence technique. This result is consistent with the value from the comparison of the Monte Carlo spectrum and the data. 


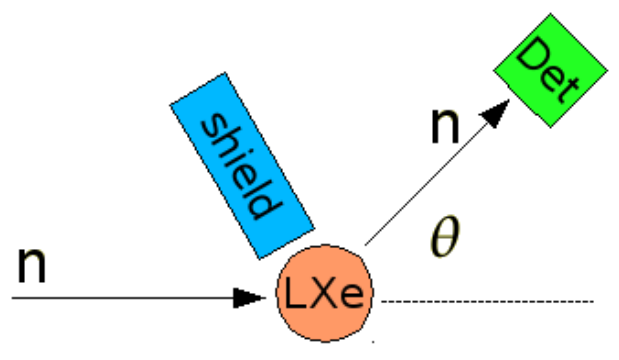

Figure 2: Principle of the measurements of the relative scintillation efficiency $\mathscr{L}_{\text {eff }}$ : The elastic energy deposition of a monoenergetic neutron beam is measured in a LXe chamber (as S1 light) and via the scattering angle. The observed amount of light relative to the gamma standard ${ }^{57} \mathrm{Co}$ is the relative scintillation efficiency.

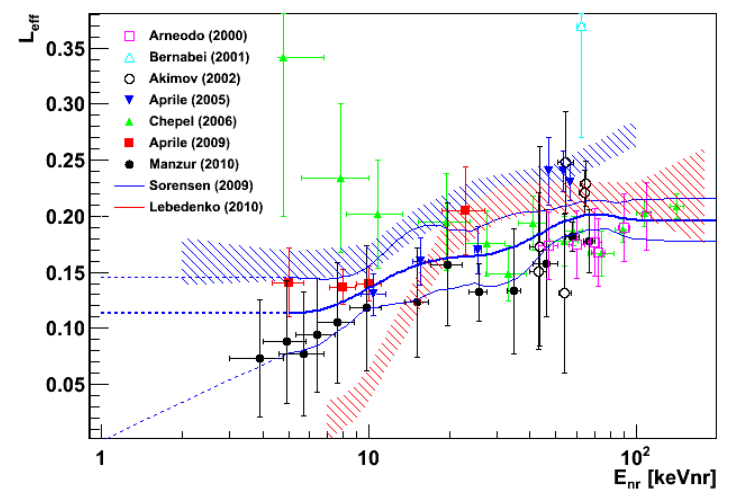

Figure 3: Compilation of all existing $\mathscr{L}_{\text {eff }}$ data [6]. The shaded regions are indirect determinations from data based on a comparison to a Monte Carlo simulation [7]. The thick line is the best fit $\mathscr{L}_{\text {eff }}$ with a constant extrapolation to lower energies. The thinner lower line is the lower $90 \%$ C.L. contour with a logarithmic extrapolation to zero.

The nuclear Recoil Energy Scale Based on the cut acceptance and the expected recoil spectrum from WIMPs, the energy region of interest for the first Dark Matter analysis has been chosen from 4-20 PE, based on the size of the prompt S1 signal. This region has to be converted to a nuclear recoil equivalent energy scale (given in keVnr) which takes into account the signal losses for nuclear recoil interactions due to quenching.

The relative scintillation efficiency $\mathscr{L}_{\text {eff }}$ of a nuclear recoil interaction of energy $E_{\text {nr }}$ is the ratio of the light yield at this energy, $\mathrm{LY}\left(E_{\mathrm{nr}}\right)$, and the light yield of the $122 \mathrm{keV}$ gamma line from ${ }^{57} \mathrm{Co}$,

$$
\mathscr{L}_{\text {eff }}\left(E_{\mathrm{nr}}\right)=\frac{\mathrm{LY}\left(E_{\mathrm{nr}}\right)}{\mathrm{LY}\left(E_{\mathrm{ee}}=122 \mathrm{keV}\right)} .
$$

In the presence of an electric field, the right hand side of the equation is modified by a factor $S_{e} / S_{n}$ to take into account field quenching. The formula allows the conversion of a light signal (measured relative to $122 \mathrm{keV}$ electron recoil equivalent energy $\left.E_{\mathrm{ee}}\right)$ to $E_{\mathrm{nr}}$. The typical measurement to determine $\mathscr{L}_{\text {eff }}$ is illustrated in Fig. 2: The true elastic energy deposition of a neutron from a monoenergetic neutron beam in a LXe chamber is measured via its scattering angle. At the same time, the scintillation light yield is measured in the LXe chamber which gives access to $\mathscr{L}_{\text {eff }}$. For lowest energies, however, the scattering angle $\theta$ is close to zero, the energy deposition in the LXe is close to the detection threshold, and the measurement becomes very difficult. Therefore, the results of various groups $[6,7]$ differ more than expected from the stated error bars, see Fig. 3. For a recent discussion of the systematics of these measurements, see [8].

In order to derive a nuclear recoil energy scale from this data, we have chosen to use all $\mathscr{L}_{\text {eff }}$ data from scattering measurements and to employ a statistical approach: The thick line in Fig. 3 is the best fit description of the data which was extrapolated to energies below $5 \mathrm{keVnr}$ using a constant function. This choice was motivated by the flat trends seen in some of the data points. The thinner lines give the upper and lower $90 \%$ C.L. contours, the lower contour was very 


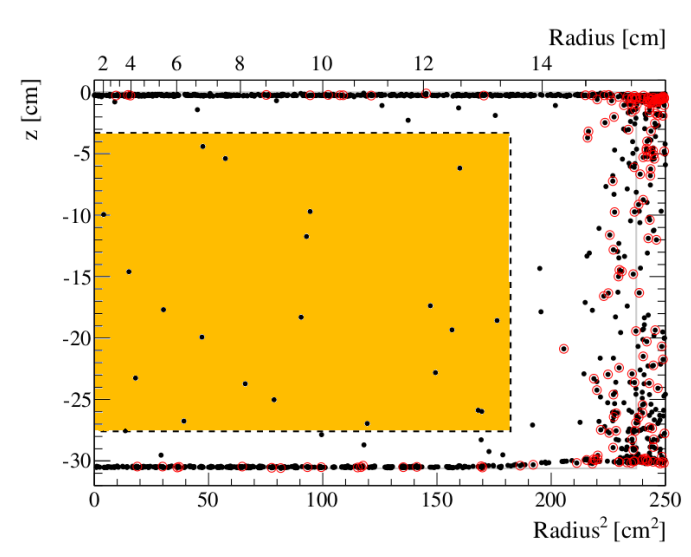

Figure 4: Distribution of the background events in the 11.2 days dataset in the energy region of interest. Most events are close to the edges of the TPC. Only 22 events end up in the fiducial volume of $40 \mathrm{~kg}$ mass which is indicated in yellow.

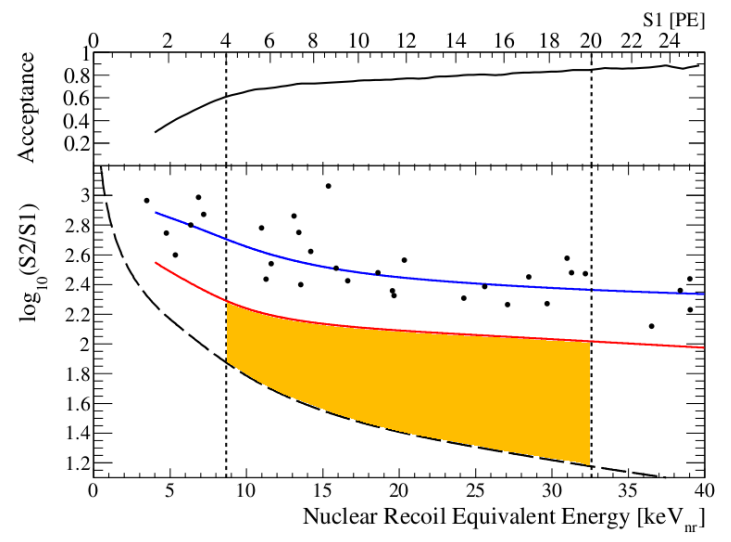

Figure 5: In the $\log _{10}(\mathrm{~S} 2 / \mathrm{S} 1)$ space, used for discrimination between signal and background, all remaining events have $\log _{10}(\mathrm{~S} 2 / \mathrm{S} 1)$ values well above the nuclear recoil median (red). No event ends up in the predefined WIMP search region (yellow).

conservatively extrapolated to zero near $1 \mathrm{keVnr}$ using a logarithmic function. In order to reflect the uncertainty currently present in $\mathscr{L}_{\text {eff }}$, we will show the results for both functions, the best fit and the lower $90 \%$ C.L. contour.

In order to clarify this situation, a new measurement of $\mathscr{L}_{\text {eff }}$ with improved systematics is currently ongoing at Columbia University. Another independent measurement is in preparation at Zurich University.

Result The data leading to the first XENON100 results were taken in stable conditions in October and November 2009. Altogether 11.2 life days of data have been used for the analysis. The data has not been blinded, however, the analysis has been performed in a quasi-blind way and all cuts and selections were developed on calibration data only.

The WIMP search region was defined to be between 4 and $20 \mathrm{PE}$, corresponding to 8.7$32.6 \mathrm{keVnr}$ (best fit $\mathscr{L}_{\text {eff }}$ ). The upper bound in $\log _{10}(\mathrm{~S} 2 / \mathrm{S} 1)$ space was the median of the nuclear recoil band from the neutron calibration, the lower a software threshold of S2 > 300 PE. A simple cylindrical fiducial volume with $40 \mathrm{~kg}$ of xenon was chosen for this analysis, and $<0.2$ background events were expected in this volume for the given exposure.

Only events with a single S2 peak (single scatter events) are selected for the WIMP analysis, since the interaction probability of WIMPs is far too small to scatter twice in the detector. Fig. 4 shows the distribution of all single scatter events in the energy region of interest; the events below the nuclear recoil median are marked in red. No nuclear recoil events are observed in the fiducial volume, indicated in yellow. As expected, the vast majority of the events is located close to the borders of the TPC. Incomplete charge (S2) collection can happen in areas very close to the PTFE walls and the field shaping electrodes of the TPC and is the typical reason for electron recoil events being shifted below the nuclear recoil median. The distribution of the remaining 22 events in the fiducial volume seems to be quite uniform, in agreement with the expectation of a background with a sizeable admixture of ${ }^{85} \mathrm{Kr}$. 


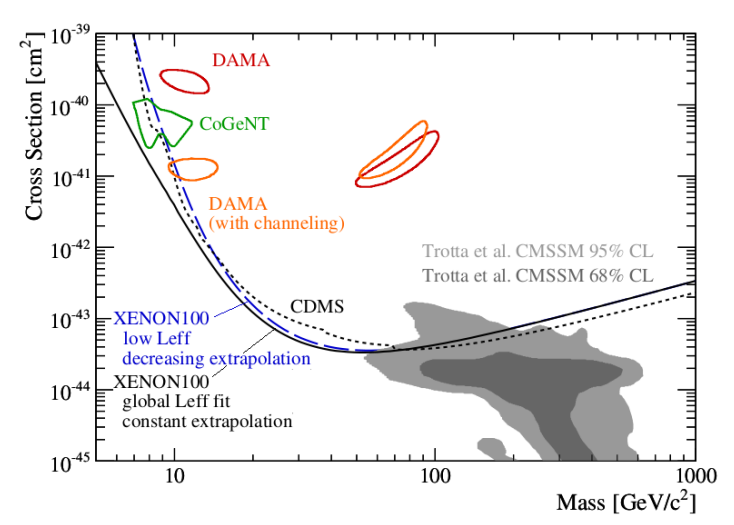

Figure 6: The limit on the spin independent WIMP-nucleon cross section derived from the first XENON100 data. The exclusion plot shows the 90\% C.L. exclusion contours for the two $\mathscr{L}_{\text {eff }}$ cases shown in Fig. 3.

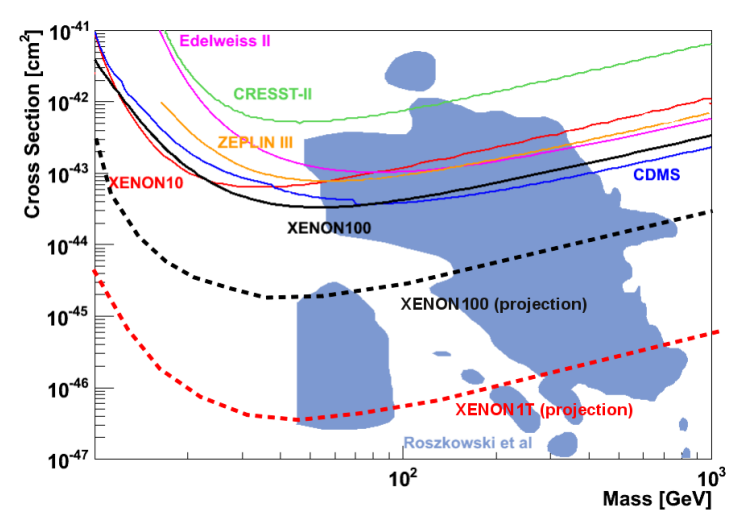

Figure 7: The sensitivity reach of XENON100 and the future project XENON1T for spin independent WIMP-nucleon couplings. XENON1T will be able to explore most of the WIMP parameter space predicted by CMSSM models.

The positions of the events remaining in the fiducial volume in $\log _{10}(\mathrm{~S} 2 / \mathrm{S} 1)$ space are shown in Fig. 5. All events are well separated from the WIMP search region. This figure is the most remarkable result of this analysis: It demonstrates that LXe detectors can be indeed used for backgroundfree WIMP searches. Due to the considerably reduced background compared to XENON10, this plot shows only a few remaining background events in the electron recoil band, where there were 100's of events (including leakage into the WIMP search region) for a similar exposure in XENON10. The upper part of Fig. 5 gives the energy dependent acceptance function not taking into account the $50 \%$ acceptance from $\log _{10}(\mathrm{~S} 2 / \mathrm{S} 1)$-based electronic recoil discrimination.

Poisson fluctuations determine the resolution at the S1 threshold and have to be taken into account to derive a limit from this data in order to treat the resolution properly. Since the expected recoil spectrum from spin-independent WIMP-nucleon scattering is a featureless falling exponential function, the resolution helps to increase the sensitivity at low WIMP masses, as more events from below the threshold fluctuate into signals above the threshold than vice versa. Fig. 6 shows the limits from this analysis, calculated for the two choices of $\mathscr{L}_{\text {eff }}$ as discussed above, assuming an isothermal WIMP halo. The acceptance-corrected exposure, weighted by a spectrum of a $100 \mathrm{GeV} / \mathrm{c}^{2}$ WIMP, is $172 \mathrm{~kg} \times$ days. For WIMP masses below $80 \mathrm{GeV} / \mathrm{c}^{2}$, this result places the lowest limit so far. At light WIMP masses, DAMA [9] and CoGeNT [10] are constrained even assuming the conservative $\mathscr{L}_{\text {eff }}$.

\section{Outlook}

This article summarizes the first Dark Matter results of XENON100, derived from 11.2 life days of data taken during a commissioning run in fall 2009 [5]. In the meantime, about $10 \times$ more science data has been acquired and a blind analysis in currently ongoing. The ultimate sensitivity of XENON100 for spin-independent WIMP-nucleon scattering, based on the background predictions from Monte Carlo simulations, is shown in Fig. 7: For 200 life days exposure and a fiducial volume of $30 \mathrm{~kg}$ mass, we expect to reach a sensitivity of $\sigma=2 \times 10^{-45} \mathrm{~cm}^{2}$ (at $100 \mathrm{GeV} / \mathrm{c}^{2}$ ). 
In order to explore the WIMP parameter space down to lower cross sections, or to confirm a possible WIMP detection, we are already in the design phase for XENON1T. This future detector will be a LXe double-phase TPC with a fiducial mass of $1000 \mathrm{~kg}$. Its expected sensitivity to spin-independent WIMP-nucleon interactions is a few $10^{-47} \mathrm{~cm}^{2}$ (see Fig. 7). A total amount of about 2.5 tons of xenon will be required for this detector. A large fiducial volume cut, together with careful material selection and LXe purification, will further decrease the background by a factor 100. The timeline for XENON1T foresees that the science phase of the projects starts already in 2014.

\section{Acknowledgments}

The author would like to thank the organizers for a very nice meeting, and N. Weiner for inventing an interesting new test to classify the results of direct Dark Matter searches.

\section{References}

[1] K. Nakamura et al. (Particle Data Group), J. Phys. G 37 (2010) 075021. and references therein.

[2] G. Bertone (ed.), Particle Dark Matter, Cambridge University Press, Cambridge 2010.

[3] K. Abe et al. (XMASS), Astropart. Phys. 31 (2009) 290.

[4] J. Angle et al. (XENON10), Phys. Rev. Lett. 100 (2008) 021303.

J. Angle et al. (XENON10), Phys. Rev. Lett. 101 (2008) 091301.

[5] E. Aprile et al. (XENON100), Phys. Rev. Lett. 105 (2010) 131302.

[6] F. Arneodo et al., Nucl. Inst. and Meth. A 449 (2000) 147.

R. Bernabei et al., EPJ direct 3 (2001) 11.

D. Akimov et al., Phys. Lett. B $\mathbf{5 2 4}$ (2002).

E. Aprile et al., Phys. Rev. D. 72 (2005).

V. Chepel et al., Astropart. Phys. 26 (2006) 58.

E. Aprile et al., Phys. Rev. C 79 (2009) 045807.

A. Manzur et al., Phys. Rev. C 81 (2010) 025808.

[7] P. Sorensen et al. (XENON10), Nucl. Inst. and Meth. A 601 (2009) 339.

V. N. Lebedenko et al. (ZEPLIN-III) Phys. Rev. D 80 (2009) 052010.

[8] A. Manalaysay, [arXiv:1007.3746].

[9] C. Savage et al., J. Cosmol. Astropart. Phys. 4 (2009) 10.

[10] C. E. Aalseth et al., [arXiv:1002.4703]. 